\title{
Yoga ist Physiotherapie bei Rückenschmerz ebenbürtig
}

\author{
Bei unspezifischen chronischen Rückenschmerzen verbessert \\ Yoga die Funktion und lindert den Schmerz. Der Effekt liegt im \\ gleichen Rahmen wie jener der Physiotherapie.
}

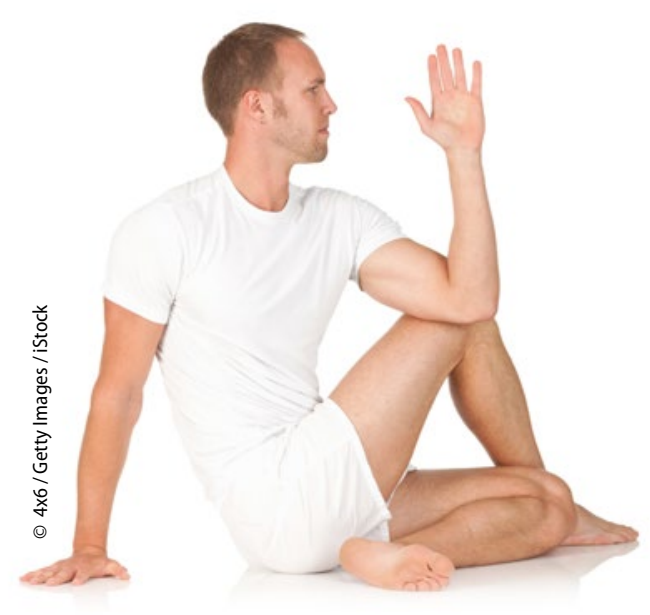

Für eine zwölfwöchige Studie wurden 479 Patienten mit chronischen Rückenschmerzen in einer mittleren Intensität von 7 auf einer Skala von 0-11 rekrutiert. Die meisten von ihnen nahmen nichtsteroidale Antirheumatika (NSAR) oder Opioide zur Schmerztherapie ein.

Randomisiert erhielten die Teilnehmer nun entweder einmal pro Woche Unterricht in Yoga, oder 15 Physiotherapiesitzungen, oder lediglich eine Edukationsbroschüre und einen Newsletter. Die Intervention lief über 12 Wochen. Primäre Endpunkte waren die Funktionseinschränkung der Lendenwirbelsäule, gemessen mit dem Roland Morris Disability Questionnaire (RMDQ), und die Schmerzintensität. Sekundäre Endpunkte waren die Einnahme von Schmerzmitteln, der globale Eindruck über eine Verbesserung der Beschwerden, die Zufriedenheit mit der Intervention und die Lebensqualität.

Die Adhärenz, das heißt die Umsetzung der erlernten Therapien im häuslichen Setting, betrug in der Yogagruppe $75 \%$ und in der Physiotherapiegruppe $64 \%$. In beiden Gruppen kam es zu einer Verbesserung der Funktionseinschrän-

\section{Hier steht eine Anzeige.}

Springer kung der Wirbelsäule. Ein signifikanter Unterschied zwischen den beiden Therapien ließ sich nicht nachweisen.

Allerdings gab es auch keinen Unterschied zwischen den Therapien und der Edukation. Die Schmerzintensität besserte sich mit Yoga um 1,7 Punkte, mit Physiotherapie um 2,3 Punkte und mit Edukation um 1,4 Punkte. Statistisch signifikant war nur der Vorteil der Physiotherapie gegenüber der Edukation.

Der therapeutische Nutzen von Yoga und Physiotherapie ließ sich auch nach einem Jahr noch nachweisen. Die Einnahme von Schmerzmitteln ging sowohl in der Yoga- als auch in der Physiotherapiegruppe um $22 \%$ stärker zurück als in der Edukationsgruppe.

- Saper RB, Lemaster C, Delitto A et al. Yoga, physical therapy, or education for chronic low back pain: a randomized noninferiority trial. Ann Intern Med. 2017;167:85-4

\section{KOMMENTAR}

Im östlichen Asien spielt die Yoga-Philosophie mit ihren Körperübungen in der Schmerztherapie eine wichtige Rolle. Diese gut geplante und durchgeführte Studie aus den USA zeigt, dass Yoga genauso wirksam ist wie Physiotherapie und dass die beiden Interventionsverfahren tendenziell besser wirksam sind als eine reine Edukation.

Die Studie krankt allerdings daran, dass nur zwei Drittel der Physiotherapiepatienten und drei Viertel der Yogapatienten die in den beiden Behandlungsgruppen unterrichtete Therapie auch tatsächlich umsetzte. Die Studie konnte leider nicht untersuchen, ob eine Kombination von Yoga, Physiotherapie und Edukation möglicherweise wirksamer ist als die drei hier untersuchten Therapieverfahren allein.

Prof. Dr. med. H.-C. Diener 